\title{
LOGARITHMIC BUMP CONDITIONS FOR CALDERÓN-ZYGMUND OPERATORS ON SPACES OF HOMOGENEOUS TYPE
}

\author{
Theresa C. Anderson, David Cruz-Uribe, SFO, \\ AND KABE MoEn
}

\begin{abstract}
We establish two-weight norm inequalities for singular integral operators defined on spaces of homogeneous type. We do so first when the weights satisfy a double bump condition and then when the weights satisfy separated logarithmic bump conditions. Our results generalize recent work on the Euclidean case, but our proofs are simpler even in this setting. The other interesting feature of our approach is that we are able to prove the separated bump results (which always imply the corresponding double bump results) as a consequence of the double bump theorem.
\end{abstract}

2010 Mathematics Subject Classification: 42B25, 42B30, $42 B 35$.

Key words: Calderón-Zygmund operators, two weight inequalities, bump conditions, spaces of homogeneous type, dyadic operators.

\section{Introduction and main results}

Given a Calderón-Zygmund singular integral $T$, the problem of finding sufficient conditions on a pair of weights $(u, \sigma)$ such that the twoweight norm inequality

$$
\int_{\mathbb{R}^{n}}|T(f \sigma)(x)|^{p} u(x) d x \leq C \int_{\mathbb{R}^{n}}|f(x)|^{p} \sigma(x) d x
$$

holds dates back to the 1970s. Significant progress has only been made in the past twenty years: for a brief history, see [10, Chapter 1]. One approach to this problem is to use the so-called $A_{p}$-bump conditions introduced by Pérez $[\mathbf{3 0}, \mathbf{3 2}]$. It was conjectured that a sufficient condition

The first author is supported by an NSF graduate fellowship. The second author is supported by the Stewart-Dorwart faculty development fund at Trinity College and by grant MTM2009-08934 from the Spanish Ministry of Science and Innovation. The third author is supported by NSF grant 1201504 . 
for (1.1) to hold is that the pair $(u, \sigma)$ satisfies

$$
\sup _{Q}\left\|u^{1 / p}\right\|_{A, Q}\left\|\sigma^{1 / p^{\prime}}\right\|_{B, Q}<\infty
$$

where the supremum is taken over all cubes in $\mathbb{R}^{n}, A$ and $B$ are Young functions that satisfy the growth conditions $\bar{A} \in B_{p^{\prime}}$ and $\bar{B} \in B_{p}$, and $\|\cdot\|$ is a normalized Orlicz norm. (For precise definitions, see below.) This problem proved quite difficult, and a number of partial results were proved $[\mathbf{8}, \mathbf{9}, \mathbf{1 2}, \mathbf{1 3}]$ before the full result was proved by Lerner $[\mathbf{2 3}]$ and by Nazarov, Reznikov, Treil, and Volberg [28] (when $p=2$ ). Much of the recent progress on this problem was due to the close connection with the $A_{2}$ conjecture on sharp one-weight norm inequalities for singular integrals - see $[\mathbf{9}]$ for details.

Recently, it was noted [14] that while the conjecture was originally stated in terms of the "double bump" condition (1.2), it was motivated by the so-called Muckenhoupt-Wheeden conjectures (see [14] and [10, Section 9.2]) and that implicit in this motivation was a weaker conjecture in terms of a pair of "separated bump" conditions: $T$ satisfies (1.1) if the pair $(u, \sigma)$ satisfy

$$
\sup _{Q}\left\|u^{1 / p}\right\|_{A, Q}\left\|\sigma^{1 / p^{\prime}}\right\|_{p^{\prime}, Q}<\infty, \quad \sup _{Q}\left\|u^{1 / p}\right\|_{p, Q}\left\|\sigma^{1 / p^{\prime}}\right\|_{B, Q}<\infty .
$$

In [14] this conjecture was proved in the special case when $A$ and $B$ are "log bumps": i.e., $A(t)=t^{p} \log (e+t)^{p-1+\delta}$ and $B(t)=t^{p^{\prime}} \log (e+$ $t)^{p^{\prime}-1+\delta}, \delta>0$. A simpler proof, one which also gives quantitative estimates on the constants for separated bumps, was found by Hytönen and Pérez $[\mathbf{2 0}]$. The exact value of the constants is important, since Hytönen [16] has shown that if the sharp constants for the separated bump condition are as conjectured, then as an immediate corollary this result yields a new proof of the sharp $A_{p}-A_{\infty}$ bounds for singular integrals $[\mathbf{1 9}, \mathbf{2 1}]$.

Remark 1.1. It has generally been accepted that the separated bump condition is weaker than the double bump condition, but no explicit pair $(u, v)$ that satisfies (1.3) but not (1.2) for a given pair of Young functions $A, B$ has appeared in the literature. We rectify this by constructing an example in Section 7 below.

The goal of this paper is to extend the double bump and separated bump results discussed above to the case of singular integrals on spaces of homogeneous type. These spaces are of interest since they often arise in applications: see for example $[\mathbf{5 , 6 , 7 , 2 7 , 3 9 ]}$. Many of the tools of classical harmonic analysis on Euclidean spaces generalize to this setting; 
nevertheless there are substantive differences and some care must be taken to insure that proofs still hold. Our arguments differ extensively from those in [14]: they have more in common with the approach taken in $[\mathbf{2 0}]$. Our proof, when restricted to the Euclidean case, is somewhat simpler than theirs, but we do not prove the same quantitative estimates on the constants. A very interesting feature of our proof is that we are able to prove the separated bump results as a consequence of the double bump estimates.

Before we can state our main results we need to make a number of definitions. By a space of homogeneous type (hereafter, SHT) we mean an ordered triple $(X, \rho, \mu)$, where $X$ is a set, $\rho$ is a quasimetric on $X$, and $\mu$ is a non-negative Borel measure on $X$ that is doubling:

$$
\mu\left(\mathscr{B}_{\rho}\left(x_{0}, 2 r\right)\right) \leq C_{d} \mu\left(\mathscr{B}_{\rho}\left(x_{0}, r\right)\right),
$$

where $\mathscr{B}_{\rho}\left(x_{0}, r\right)=\left\{x \in X: \rho\left(x, x_{0}\right)<r\right\}$. The smallest such constant $C_{d}$ is called the doubling constant of $\mu$. We also assume that $\mu$ is non-trivial, i.e., for every ball, $0<\mu\left(\mathscr{B}_{\rho}\left(x_{0}, r\right)\right)<\infty$. For further details, see $[\mathbf{5}]$ or $[\mathbf{6}]$.

Remark 1.2. For brevity, hereafter we will say that a constant depends on $X$ and write $C(X, \ldots)$ if the constant depends on the triple $(X, \rho, \mu)$.

A function $K: X \times X \backslash\{(x, x)\} \rightarrow \mathbb{R}$ is a Calderón-Zygmund kernel if there exist $\eta>0$ and $C<\infty$ such that for all $x_{0} \neq y \in X$ and $x \in X$ it satisfies the decay condition:

$$
\left|K\left(x_{0}, y\right)\right| \leq \frac{C}{\mu\left(\mathscr{B}_{\rho}\left(x_{0}, \rho\left(x_{0}, y\right)\right)\right)}
$$

and the smoothness condition: for $\rho\left(x_{0}, x\right) \leq \eta \rho\left(x_{0}, y\right)$,

$$
\begin{aligned}
\left|K(x, y)-K\left(x_{0}, y\right)\right|+\mid K(y, x) & -K\left(y, x_{0}\right) \mid \\
\leq & C\left(\frac{\rho\left(x, x_{0}\right)}{\rho\left(x_{0}, y\right)}\right)^{\eta} \frac{1}{\mu\left(\mathscr{B}_{\rho}\left(x_{0}, \rho\left(x_{0}, y\right)\right)\right)} .
\end{aligned}
$$

An operator $T$ is associated with a Calderón-Zygmund kernel $K$ if for every $f \in C_{c}(X)$,

$$
T f(x)=\int_{X} f(y) K(x, y) d \mu(y), \quad x \notin \operatorname{supp}(f) .
$$

If $T$ is bounded on $L^{2}(X, \mu)$, then $T$ is referred to as a Calderón-Zygmund operator. 
The bump conditions discussed above are given in terms of Orlicz norms. Here we summarize some of the basic properties we need; for the general theory of Orlicz spaces, see [35] or [10, Chapter 5]. A Young function is a continuous, convex, increasing function $A:[0, \infty) \rightarrow[0, \infty)$ such that $A(0)=0$ and $A(t) / t \rightarrow \infty$ as $t \rightarrow \infty$. It is often convenient to assume that $A(1)=1$ but this is not strictly necessary. Note that $A(t)=t$ is not a Young function though $t^{p}$ is for $p>1$. However, in many cases results for Young functions hold in this limiting case. The Young functions we are interested in are referred to as log bumps: $A(t)=t^{p} \log (e+t)^{p-1+\delta}, \delta>0$.

Given two Young functions $A$ and $B$, we write that $A \lesssim B$ if there exists constants $c, t_{0}>0$ such that $A(t) \leq B(c t)$ for all $t \geq t_{0}$. Note that given any Young function $A, t \lesssim A(t)$. We will write $A \approx B$ if there exists $c_{1}, c_{2}, t_{0}>0$ such that $c_{1} A(t) \leq B(t) \leq c_{2} A(t)$ for all $t \geq t_{0}$.

Given a Young function $A$ and a set $E$ such that $0<\mu(E)<\infty$, define the Orlicz space norm

$$
\|v\|_{A, E}=\inf \left\{\lambda>0: f_{E} A\left(\frac{|v(x)|}{\lambda}\right) d \mu(x) \leq 1\right\},
$$

where $f_{E}=\mu(E)^{-1} \int_{E}$. If $A(t)=t^{p}, 1 \leq p<\infty$, then

$$
\|v\|_{A, E}=\left(f_{E}|v|^{p} d \mu\right)^{1 / p}=\|v\|_{p, E} .
$$

If $A \lesssim B$, then there exists a constant $C$ such that $\|f\|_{A, E} \leq C\|f\|_{B, E}$.

Given a Young function $A$, define $\bar{A}$, the complementary function, by

$$
\bar{A}(t)=\sup _{s>0}\{s t-A(s)\} .
$$

It can be shown that $\bar{A}$ is also a Young function. Given $A$, we have the generalized Hölder's inequality: for any set $E, 0<\mu(E)<\infty$,

$$
f_{E}|f(x) g(x)| d \mu(x) \leq 2\|f\|_{A, E}\|g\|_{\bar{A}, E} .
$$

Denote the inverse of a Young function by $A^{-1}$. More generally, given three Young functions $A, B, C$ such that

$$
B^{-1}(t) C^{-1}(t) \leq c A^{-1}(t),
$$

then there exists a constant $K$ such that

$$
\|f g\|_{A, E} \leq K\|f\|_{B, E}\|g\|_{C, E} .
$$


Given $1<p<\infty$ we define the class $B_{p}$ : a Young function $A \in B_{p}$ if

$$
[A]_{B_{p}}=\int_{1}^{\infty} \frac{A(t)}{t^{p}} \frac{d t}{t}<\infty .
$$

In the special case of $\log$ bumps, if $A(t)=t^{p} \log (e+t)^{p-1+\delta}$ then $\bar{A}(t) \approx$ $t^{p^{\prime}} \log (e+t)^{-1-\left(p^{\prime}-1\right) \delta}$, and so $\bar{A} \in B_{p^{\prime}}$.

We can now define our bump conditions. Given Young functions $A$ and $B$, and a pair of weights $(u, \sigma)$, define

$$
[u, \sigma]_{A, B, p}=\sup _{\mathscr{B}_{\rho}}\left\|u^{1 / p}\right\|_{A, \mathscr{B}_{\rho}}\left\|\sigma^{1 / p^{\prime}}\right\|_{B, \mathscr{B}_{\rho}},
$$

and

$$
[u, \sigma]_{A, p}=\sup _{\mathscr{B}_{\rho}}\left\|u^{1 / p}\right\|_{A, \mathscr{B}_{\rho}}\left\|\sigma^{1 / p^{\prime}}\right\|_{p^{\prime}, \mathscr{B}_{\rho}}
$$

where the suprema are taken over all balls $\mathscr{B}_{\rho}$ in $X$. Note that by symmetry we have that if $B$ is another Young function, then

$$
[\sigma, u]_{B, p^{\prime}}=\sup _{\mathscr{B}_{\rho}}\left\|u^{1 / p}\right\|_{p, \mathscr{B}_{\rho}}\left\|\sigma^{1 / p^{\prime}}\right\|_{B, \mathscr{B}_{\rho}}
$$

By weights $u$ and $\sigma$ we always mean non-negative measurable functions on $X$ that are finite almost everywhere and positive on sets of positive measure. Many authors assume that weights are locally integrable; however, when working with bump conditions this assumption can be avoided by an approximation argument. As was shown in [10, Section 7.2], we can always assume that $u$ and $\sigma$ are bounded and bounded away from 0 on $X$, provided that in the norm inequality being proved we are working with a function $f \in \cap_{p>1} L^{p}(X, \mu)$ : for example, $f$ is a bounded function of compact support.

Remark 1.3. Since bounded functions of compact support are dense in any weighted space $L^{p}(X, u)$, we will hereafter assume that $u, \sigma$, and $f$ satisfy these conditions. Moreover, since $T$ is linear we will also assume without loss of generality that $f$ is non-negative.

We can now state our main results. The first generalizes the double bump condition to SHT.

Theorem 1.4. Given an $\operatorname{SHT}(X, \rho, \mu)$, suppose the pair of weights $(u, \sigma)$ satisfies $[u, \sigma]_{A, B, p}<\infty$, where $\bar{A} \in B_{p^{\prime}}$ and $\bar{B} \in B_{p}$. Then a CalderónZygmund operator $T$ satisfies the strong type inequality

$$
\|T(f \sigma)\|_{L^{p}(u)} \leq C(T, X)[u, \sigma]_{A, B, p}[\bar{A}]_{B_{p^{\prime}}}^{1 / p^{\prime}}[\bar{B}]_{B_{p}}^{1 / p}\|f\|_{L^{p}(\sigma)} .
$$

The next two results give separated bump conditions for weak and strong type inequalities. 
Theorem 1.5. Given an $S H T(X, \rho, \mu)$, suppose the pair of weights $(u, \sigma)$ is such that $[u, \sigma]_{A, p}<\infty$, where $A(t)=t^{p} \log (e+t)^{p-1+\delta}$. Then a Calderón-Zygmund operator $T$ satisfies the weak type inequality

$$
\|T(f \sigma)\|_{L^{p, \infty}(u)} \leq C(T, X, p, \delta)[u, \sigma]_{A, p}\|f\|_{L^{p}(\sigma)} .
$$

Theorem 1.6. Given an $S H T(X, \rho, \mu)$, suppose the pair of weights $(u, \sigma)$ is such that $[u, \sigma]_{A, p}<\infty$ and $[\sigma, u]_{B, p^{\prime}}<\infty$, where $A(t)=t^{p} \log (e+$ $t)^{p-1+\delta}$ and $B(t)=t^{p^{\prime}} \log (e+t)^{p^{\prime}-1+\delta}$. Then a Calderón-Zygmund operator $T$ satisfies the strong type inequality

$$
\|T(f \sigma)\|_{L^{p}(u)} \leq C(T, X, p, \delta)\left([u, \sigma]_{A, p}+[\sigma, u]_{B, p^{\prime}}\right)\|f\|_{L^{p}(\sigma)} .
$$

The remainder of this paper is organized as follows. In Section 2 we introduce the powerful notion of dyadic grids on spaces of homogeneous type. These were first constructed by Christ [5], but we will follow the more recent work of Hytönen and Kairema [17]. These grids let us naturally extend the Calderón-Zygmund decomposition and the techniques of the so-called sparse operators to an SHT. In Section 3 we will reduce the proof of our main theorems to proving estimates for sparse operators. The proof depends on results that in the Euclidean case are due to Lerner [23] and Lacey, Sawyer, and Uriarte-Tuero [22]. We give the corresponding results for an SHT. In Section 4 we prove Theorem 1.4 by proving the corresponding result for sparse operators. The proof is nearly identical to the proof given in [9] in the Euclidean case, so we only sketch the details. In Section 5 we prove a weak $(1,1)$ inequality for sparse operators that we need for our proof of Theorem 1.5. Our proof follows the broad outline of the analogous result for singular integrals in Euclidean spaces due to Pérez [31]; however, it is simpler because of the localized behavior of sparse operators. In Section 6 we prove Theorems 1.5 and 1.6. Finally, in Section 7 we construct a pair of weights on the real line that satisfies a separated logarithmic bump condition but not the corresponding double bump condition.

In our proofs of Theorems 1.5 and 1.6 the only place we use that $A$ and $B$ are $\log$ bumps is in the final argument in Section 6 . However, despite repeated efforts we are unable to eliminate this assumption. Nevertheless, we conjecture that both results are true with the weaker assumption that $\bar{A} \in B_{p^{\prime}}$ and $\bar{B} \in B_{p}$, but we believe that new techniques will be required to prove this. On the other hand, very recently Nazarov, Reznikov, and Volberg [29] have given a proof of the separated bump result in Euclidean spaces using Bellman functions. Certain aspects of their proof lead them to suggest that the full conjecture may be false. 
Acknowledgement. The authors would like to thank the anonymous referee for his or her detailed comments and suggestions.

\section{Dyadic cubes in spaces of homogeneous type}

An important tool for our proofs is the concept of a dyadic grid $\mathscr{D}$ on an SHT and the concept of a sparse family $S$ in $\mathscr{D}$. These generalize the classical Calderón-Zygmund decomposition (cf. [10, Appendix A]). The following result is due to Hytönen and Kairema [17] (see also [5]).

Theorem 2.1. Given an $\operatorname{SHT}(X, \rho, \mu)$, there exist constants $C>0,0<$ $\eta, \epsilon<1$, depending on $X$, a family of sets $\mathscr{D}=\cup_{k \in \mathbb{Z}} \mathscr{D}_{k}$ (called a dyadic decomposition of $X)$, and a corresponding family of points $\left\{x_{c}(Q)\right\}_{Q \in \mathscr{D}}$ that satisfy the following properties:

(1) for all $k \in \mathbb{Z}$, the cubes in $\mathscr{D}_{k}$ are pairwise disjoint and $X=$ $\bigcup_{Q \in \mathscr{D}_{k}} Q$

(2) if $Q_{1}, Q_{2} \in \mathscr{D}$ then either $Q_{1} \cap Q_{2}=\emptyset, Q_{1} \subset Q_{2}$, or $Q_{2} \subset Q_{1}$;

(3) for any $Q_{1} \in \mathscr{D}_{k}$ there exists at least one $Q_{2} \in \mathscr{D}_{k+1}$ (called a child of $Q_{1}$ ) such that $Q_{2} \subset Q_{1}$, and there exists exactly one $Q_{3} \in \mathscr{D}_{k-1}$ (called the parent of $Q_{1}$ ) such that $Q_{1} \subset Q_{3}$;

(4) if $Q_{2}$ is a child of $Q_{1}$, then $\mu\left(Q_{2}\right) \geq \epsilon \mu\left(Q_{1}\right)$;

(5) $\mathscr{B}_{\rho}\left(x_{c}(Q), \eta^{k}\right) \subset Q \subset \mathscr{B}_{\rho}\left(x_{c}(Q), C \eta^{k}\right)$.

Remark 2.2. The number of children contained in a given dyadic cube is uniformly bounded. It follows from (1) and (4) above that this number is bounded by $1 / \epsilon$.

The sets $Q \in \mathscr{D}$ are referred to as dyadic cubes with center $x_{c}(Q)$ and sidelength $\eta^{k}$, but we must emphasize that these are not cubes in any standard sense even if the underlying space is $\mathbb{R}^{n}$, and care must be taken when visualizing them. An exact characterization of the kinds of sets which can be dyadic cubes is given in [18]. Below we will need the dilations $\lambda Q, \lambda>1$, of dyadic cubes. However, these will actually be balls containing $Q$ : given a cube $Q$, we define

$$
\lambda Q=\mathscr{B}_{\rho}\left(x_{c}(Q), \lambda C \eta^{k}\right) .
$$

Families of dyadic grids can be constructed that have additional useful properties: see [17]. We apply one such family to show that our bump conditions can be restated in terms of dyadic cubes. Given a dyadic 
grid $\mathscr{D}$, a pair of weights $(u, \sigma)$, and a Young function $A$, define

$$
[u, \sigma]_{A, p}^{\mathscr{D}}=\sup _{Q \in \mathscr{D}}\left\|u^{1 / p}\right\|_{A, Q}\left\|\sigma^{1 / p^{\prime}}\right\|_{p^{\prime}, Q} .
$$

We define $[u, \sigma]_{A, B, p}^{\mathscr{D}}$ similarly.

Lemma 2.3. Given a pair of weights $(u, \sigma)$, and Young functions $A$ and $B$,

$$
[u, \sigma]_{A, p} \approx \sup _{\mathscr{D}}[u, \sigma]_{A, p}^{\mathscr{D}}, \quad[u, \sigma]_{A, B, p} \approx \sup _{\mathscr{D}}[u, \sigma]_{A, B, p}^{\mathscr{D}} .
$$

In both cases, the constants in the equivalence depend only on $X$.

Proof: We prove the first equivalence; the proof of the second is identical. Given a dyadic grid $\mathscr{D}$ and $Q \in \mathscr{D}$, by Theorem 2.1 there exists a ball $\mathscr{B}_{\rho}$ such that $Q \subset \mathscr{B}_{\rho}$ and $\mu\left(\mathscr{B}_{\rho}\right) \approx \mu(Q)$. Therefore, there exists $C(X)>1$ such that for any $\lambda>0$,

$$
\begin{aligned}
f_{Q} A\left(\frac{u(x)}{\lambda}\right) d \mu(x) & \leq C(X) f_{\mathscr{B}_{\rho}} A\left(\frac{u(x)}{\lambda}\right) d \mu(x) \\
& \leq f_{\mathscr{B}_{\rho}} A\left(\frac{C(X) u(x)}{\lambda}\right) d \mu(x) ;
\end{aligned}
$$

the last inequality holds since Young functions are convex. Hence, by the definition of the Orlicz norm, $\|u\|_{A, Q} \leq C(X)\|u\|_{A, \mathscr{B}_{\rho}}$. The same estimate holds for the norm of $\sigma$. We thus have that

$$
\sup _{\mathscr{D}}[u, \sigma]_{A, p}^{\mathscr{D}} \leq C(X)[u, \sigma]_{A, p} .
$$

To prove the reverse inequality, we use the fact that there exists a family of dyadic grids $\mathscr{D}^{1}, \ldots, \mathscr{D}^{J}, J$ depending only on $X$, that satisfy the properties of Theorem 2.1 with the additional property that given any ball $\mathscr{B}_{\rho}$, there exists $j$ and $Q \in \mathscr{D}^{j}$ such that $\mathscr{B}_{\rho} \subset Q$ and $\mu\left(\mathscr{B}_{\rho}\right) \approx \mu(Q)$. (See [17, Theorem 4.1].) Therefore, we can repeat the above argument, reversing the roles of $\mathscr{B}_{\rho}$ and $Q$, to get

$$
[u, \sigma]_{A, p} \leq C(X) \sup _{\mathscr{D}}[u, \sigma]_{A, p}^{\mathscr{D}} .
$$

Given a collection of dyadic cubes $\mathscr{D}$, a sparse family $S \subset \mathscr{D}$ is a collection of dyadic cubes for which there exists a collection of sets $\{E(Q)$ : $Q \in S\}$ such that the sets $E(Q)$ are pairwise disjoint, $E(Q) \subset Q$, and $\mu(Q) \leq 2 \mu(E(Q))$. Sparse families of cubes are a generalization of the Calderón-Zygmund decomposition in the Euclidean case. Using Theorem 2.1 we can form this decomposition in an SHT. In order to do this we need the Lebesgue differentiation theorem, which holds in any SHT. 
This fact seems to be new, though the proof only consists of assembling pieces already present in the literature: in particular, it is implicit in $[37]$.

Lemma 2.4. Given an SHT $(X, \rho, \mu)$, the Lebesgue differentiation theorem holds: for $\mu$-almost every $x \in X$,

$$
\lim _{r \rightarrow 0} \frac{1}{\mu\left(\mathscr{B}_{\rho}(x, r)\right)} \int_{\mathscr{B}_{\rho}(x, r)}|f(y)-f(x)| d \mu(y)=0 .
$$

Proof: Macías and Segovia, building on their earlier work in [25], showed in $[26]$ that given any $\operatorname{SHT}(X, \rho, \mu)$, there exists an equivalent quasidistance $\delta$ (i.e., there exist constants $c_{1}, c_{2}$ depending on $X$ such that for all $\left.x, y \in X, c_{1} \rho(x, y) \leq \delta(x, y) \leq c_{2} \rho(x, y)\right)$, such that given any ball $\mathscr{B}_{\delta}$ with respect to $\delta$, then $\left(\mathscr{B}_{\delta}, \delta, \mu\right)$ is again a space of homogeneous type, and the constants are independent of the ball $\mathscr{B}_{\delta}$.

Toledano [37] proved that since $\mu\left(\mathscr{B}_{\delta}\right)<\infty$, the measure $\mu$ when restricted to $\mathscr{B}_{\delta}$ is regular. The Lebesgue differentiation theorem holds for regular measures: this follows from the standard argument (cf. [36, Chapter 7]) using the fact that the maximal operator is weak $(1,1)$ on $L^{1}\left(\mathscr{B}_{\delta}, \mu\right)[\mathbf{5}]$ and that smooth functions of compact support are dense in $L^{1}\left(\mathscr{B}_{\delta}, \mu\right)[\mathbf{3 6}$, Chapter 3]. Therefore, we have that for $\mu$-almost every $x \in \mathscr{B}_{\delta}$,

$$
\lim _{r \rightarrow 0} f_{\mathscr{B}_{\delta}(x, r)}|f(y)-f(x)| d \mu(y)=0 .
$$

Since $\rho$ and $\delta$ are equivalent and $\mu$ is doubling, it follows immediately that (2.2) holds in $\mathscr{B}_{\rho}$. Since $X$ can be covered by a countable collection of $\rho$-balls, it holds for $\mu$-almost every $x \in X$.

Remark 2.5. As a corollary to this proof we also have that $C_{c}(X)$ is dense in $L^{1}(X, \mu)$. This fact, together with Lemma 2.4, can be used to simplify the hypotheses for results in a number of papers: see, for example, $[\mathbf{3}, \mathbf{4}]$.

Corollary 2.6. Given an $\operatorname{SHT}(X, \rho, \mu)$ and a dyadic grid $\mathscr{D}$ that satisfies the hypotheses of Theorem 2.1, then for $\mu$-almost every $x \in X$, if $\left\{Q_{k}\right\}$ is the sequence of dyadic cubes in $\mathscr{D}$ such that $\cap_{k} Q_{k}=\{x\}$, then

$$
\lim _{k \rightarrow \infty} f_{Q_{k}}|f(y)-f(x)| d \mu(y)=0 .
$$

Proof: First note that since $\rho$ is a quasi-distance and $\mu$ is doubling, if $x \in \mathscr{B}_{\rho}\left(x_{0}, r\right)$, then there exists a constant $K$ such that $\mathscr{B}_{\rho}\left(x_{0}, r\right) \subset$ 
$\mathscr{B}_{\rho}(x, 2 K r)$ and $\mu\left(\mathscr{B}_{\rho}(x, 2 K r)\right) \approx \mu\left(\mathscr{B}_{\rho}\left(x_{0}, r\right)\right)$. Hence,

$$
f_{\mathscr{B}_{\rho}\left(x_{0}, r\right)}|f(y)-f(x)| d \mu(y) \leq C f_{\mathscr{B}_{\rho}(x, 2 K r)}|f(y)-f(x)| d \mu(y) .
$$

Therefore, if $\mathscr{B}_{k}$ is a sequence of balls such that $\bigcap_{k} \mathscr{B}_{k}=\{x\}$, then it follows from Lemma 2.4 that

$$
\lim _{k \rightarrow \infty} f_{\mathscr{B}_{k}}|f(y)-f(x)| d \mu(y)=0 .
$$

Now for any $k$, by Theorem 2.1 there exists a ball $\mathscr{B}_{k}$ such that $x \in$ $Q_{k} \subset \mathscr{B}_{k}$ and $\mu\left(\mathscr{B}_{k}\right) \leq C \mu\left(Q_{k}\right)$. Then (2.3) follows at once from (2.4).

Remark 2.7. Corollary 2.6 was stated in [2] without proof and with a reference to $[\mathbf{3 7}]$. However, as we noted, this result was only implicit there.

We now extend the Calderón-Zygmund decomposition to an SHT. We give a version that holds for Orlicz norms and not just for $L^{1}$ averages. We begin by defining a dyadic Orlicz maximal operator. Given a dyadic grid $\mathscr{D}$ and a Young function $\Phi$, define

$$
M_{\Phi}^{\mathscr{D}} f(x)=\sup _{x \in Q \in \mathscr{D}}\|f\|_{\Phi, Q}
$$

The standard dyadic maximal operator is gotten by taking $\Phi(t)=t$ (which is not a Young function); in this case we simply write $M^{\mathscr{D}}$.

Theorem 2.8. Given an $\operatorname{SHT}(X, \rho, \mu)$ such that $\mu(X)=\infty$, a dyadic grid $\mathscr{D}$, and a Young function $\Phi$, suppose that $f$ is a measurable function such that $\|f\|_{\Phi, Q} \rightarrow 0$ as $\mu(Q) \rightarrow \infty$. Then the following are true:

(1) For each $\lambda>0$, there exists a collection $\left\{Q_{j}\right\} \subset \mathscr{D}$ that is pairwise disjoint, maximal with respect to inclusion, and such that

$$
\Omega_{\lambda}=\left\{x \in X: M_{\Phi}^{\mathscr{D}} f(x)>\lambda\right\}=\bigcup_{j} Q_{j} .
$$

Moreover, there exists a constant $C(X)$ such that for every $j$,

$$
\lambda<\|f\|_{\Phi, Q_{j}} \leq C(X) \lambda .
$$

(2) Given $a>2 / \epsilon$, where $\epsilon$ is as in Theorem 2.1, for each $k \in \mathbb{Z}$ let $\left\{Q_{j}^{k}\right\}_{j}$ be the collection of maximal dyadic cubes in (1) with

$$
\Omega_{k}=\left\{x \in X: M_{\Phi}^{\mathscr{D}} f(x)>a^{k}\right\}=\bigcup_{j} Q_{j}^{k} .
$$

Then the set of cubes $S=\left\{Q_{j}^{k}\right\}$ is sparse, and $E\left(Q_{j}^{k}\right)=Q_{j}^{k} \backslash \Omega_{k+1}$. 
If $\mu(X)<\infty$, then (1) holds provided that $\lambda>f_{X}|f(x)| d \mu(x)$, and (2) holds for all $k$ such that $a^{k}>f_{X}|f(x)| d \mu(x)$.

The proof of Theorem 2.8 is essentially identical to that in the Euclidean in case: see, for example, [10, Appendix A.1]. The constant $C(X)$ in (1) depends on the doubling constant of $\mu$. When $\mu(X)<\infty$ some minor modifications to the proof are necessary; these correspond to what is often referred to as a "local" Calderón-Zygmund decomposition. To make them it suffices to note that in this case $X$ is bounded (see [15]). Therefore, by Theorem 2.1, for all dyadic cubes $Q$ sufficiently large, $X=Q$, and so the argument for (1) still holds if we take $\lambda>f_{X}|f(x)| d \mu(x)=f_{Q}|f(x)| d \mu(x)$.

Theorem 2.9. Given an $S H T(X, \rho, \mu)$ such that $\mu(X)=\infty$, and a dyadic grid $\mathscr{D}$, suppose $f$ is a function such that $\|f\|_{1, Q} \rightarrow 0$ as $\mu(Q) \rightarrow$ $\infty$. Then for any $\lambda>0$ there exists a pairwise disjoint family $\left\{Q_{j}\right\} \subset \mathscr{D}$ and functions $b$ and $g$ such that:

(1) $f=b+g$;

(2) $g=f \chi_{\left\{x: M^{\mathscr{D}} f(x) \leq \lambda\right\}}+\sum_{j} f_{Q_{j}}$;

(3) for $\mu$-a.e. $x \in X,|g(x)| \leq C(X) \lambda$;

(4) $b=\sum_{j} b_{j}$, where $b_{j}=\left(f-f_{Q_{j}}\right) \chi_{Q_{j}}$;

(5) $\operatorname{supp}\left(b_{j}\right) \subset Q_{j}$ and $f_{Q_{j}} b_{j}(x) d \mu(x)=0$.

If $\mu(X)<\infty$, then this decomposition still exists if we take $\lambda>$ $f_{X}|f(x)| d \mu(x)$.

Theorem 2.9 is proved exactly as in the Euclidean case, taking $\left\{Q_{j}\right\}$ to be the cubes from (1) in Theorem 2.8. The proof that $g \in L^{\infty}$ requires the Lebesgue differentiation theorem, Corollary 2.6.

\section{Reduction to estimates for sparse operators}

Given a dyadic grid $\mathscr{D}$ and a sparse family $S$ in $\mathscr{D}$, define the sparse operator $T^{S}=T^{S, \mathscr{D}}$ by

$$
T^{S} f(x)=\sum_{Q \in S}\left(f_{Q} f d \mu\right) \cdot \chi_{Q}(x) .
$$

The operator $T^{S}$ is a positive, dyadic Calderón-Zygmund operator. It follows from the definition of sparseness that $T^{S}$ is bounded on $L^{2}(\mu)$ and satisfies a weak $(1,1)$ inequality: see [2, Lemmas 6.4 and 6.5].

A key feature of our proofs is that we reduce the problem for CalderónZygmund operators to proving the same estimates for sparse operators. To do so we need to extend two results from the Euclidean setting to 
spaces of homogeneous type. The first result is due to Lerner [23] in the Euclidean setting; it was central to his greatly simplified proof of the $A_{2}$ conjecture and strong bump conjecture. We defer the proof until the end of this section.

Theorem 3.1. Given an SHT $(X, \rho, \mu)$ and a Calderón-Zygmund operator $T$, then for any Banach function space $Y$,

$$
\|T(f \sigma)\|_{Y} \leq C(X, T) \sup _{\mathscr{D}, S}\left\|T^{S}(f \sigma)\right\|_{Y},
$$

where the supremum is taken over every dyadic grid $\mathscr{D}$ in $(X, \rho, \mu)$ and over every sparse family $S$ in $\mathscr{D}$.

By taking $Y$ equal to $L^{p, \infty}(u)$ or $L^{p}(u)$, it follows immediately from Theorem 3.1 that to prove estimates for Calderón-Zygmund operators, it suffices to prove them for all sparse operators $T^{S}$ with constants independent of $S$ and $\mathscr{D}$. Below, we will prove Theorems 1.4 and 1.5 by establishing such estimates.

To prove Theorem 1.6 we need to argue indirectly using a result which connects the weak and strong type norm inequalities of sparse operators. In the Euclidean case this theorem is due to Lacey, Sawyer, and Uriarte-Tuero [22].

Theorem 3.2. Given an $\operatorname{SHT}(X, \rho, \mu)$, let $\mathscr{D}$ be a dyadic grid and $S$ a sparse family in $\mathscr{D}$. Then

$$
\begin{aligned}
\left\|T^{S}(\cdot \sigma)\right\|_{L^{p}(\sigma) \rightarrow L^{p}(u)} \approx\left\|T^{S}(\cdot \sigma)\right\|_{L^{p}(\sigma) \rightarrow L^{p, \infty}(u)} & \\
& +\left\|T^{S}(\cdot u)\right\|_{L^{p^{\prime}}(u) \rightarrow L^{p^{\prime}, \infty}(\sigma)} .
\end{aligned}
$$

The constants in the equivalence depend only on $X, T$, and $p$; in particular they are independent of $\mathscr{D}$ and $S$.

The proof of Theorem 3.2 passes through the equivalence of the weak and strong type inequalities to certain testing conditions. The proof of this equivalence for weak type inequalities in an SHT is the same as in [22, Section 2.2] in the Euclidean setting; it is straightforward to see that the only properties of dyadic cubes used in the proof are the those given in Theorem 2.1. The proof of this equivalence for strong type inequalities in [22] is much more involved; however, a simpler proof was recently given by Treil [38] and as he notes (see Section 5 of his paper), this proof also extends to an SHT with essentially no change.

Given Theorem 3.2, Theorem 1.6 follows from the characterization of the weak type inequality in Theorem 1.5. We will make this precise in Section 6 below. 
Proof of Theorem 3.1: Our proof draws heavily on the results proved in $[2]$ and we refer the reader there for complete details.

By our assumptions on $f$ and $\sigma$ we can, for clarity, replace $f \sigma$ by $f$. As was proved in [17, Proposition 4.3], if we fix a point $x_{0} \in X$, we can construct a dyadic grid $\mathscr{D}^{*}$ satisfying Theorem 2.1 that contains a sequence of nested dyadic cubes $\left\{Q_{N}\right\}$ such that $x_{0}$ is the center of each cube $Q_{N}$ and such that $\bigcup_{N} Q_{N}=X$. Therefore, by duality and Fatou's Lemma, there exists $g$ in the associate space $Y^{\prime},\|g\|_{Y^{\prime}}=1$, such that

$$
\|T f\|_{Y}=\int_{X}|T f(x)| g(x) d \mu(x) \leq \liminf _{N \rightarrow \infty} \int_{Q_{N}}|T f(x)| g(x) d \mu(x) .
$$

Fix $N>0$; we will prove that the final integral is bounded by $C$ sup $\left\|T^{S} f\right\|_{Y}$, where the supremum is taken over some collection of $S$ and $\mathscr{D}$, but the constant is independent of these and also independent of $N$.

As was proved in $\left[\mathbf{2}\right.$, Section 5], there exist $C_{1}, C_{2}, \eta>0$ such that for $\mu$-a.e. $x \in Q_{N}$,

$$
|T f(x)| \leq C_{1} M f(x)+C_{2} \sum_{i=1}^{\infty} \frac{1}{2^{i \eta}} A_{i} f(x),
$$

where

$$
A_{i} f(x)=\sum_{Q \in S_{N}} f_{2^{i} Q} f(y) d \mu(y) \cdot \chi_{Q}(x)
$$

and $S_{N}$ is a sparse subset of $\mathscr{D}^{*}$ that consists of dyadic sub-cubes of $Q_{N}$. The constants depend only on $X$ and $T$; in particular $C_{1}$ depends on the fact (see [5]) that $T: L^{1}(X, \mu) \rightarrow L^{1, \infty}(X, \mu)$. Therefore, by Hölder's inequality (with respect to $Y$ and $Y^{\prime}$ ),

$\int_{Q_{N}}|T f(x)| g(x) d \mu(x) \leq C_{1}\left\|M f \cdot \chi_{Q_{N}}\right\|_{Y}+C_{2} \sum_{i} 2^{-i \eta}\left\|A_{i} f \cdot \chi_{Q_{N}}\right\|_{Y}=I_{1}+I_{2}$.

To estimate $I_{1}$ we give a pointwise estimate for $M f(x)$. By $[\mathbf{1 7}$, Theorem 7.9] there exists a constant $K=K(X)$ and a collection $\mathscr{D}^{1}, \ldots, \mathscr{D}^{K}$ of dyadic grids such that for every $x \in X$,

$$
M f(x) \leq C(X) \sum_{k=1}^{K} M^{k} f(x)
$$

where $M^{k}=M^{\mathscr{D}^{k}}$ is the dyadic maximal operator defined with respect to $\mathscr{D}^{k}$. We claim that for each $k$ there exists a sparse subset $S_{k}$ (depending on $f$ ) such that

$$
M^{k} f(x) \leq C(X) T^{S_{k}} f(x) .
$$


This follows from (2) in Theorem 2.8. With the notation of this result, let $S_{k}=\left\{Q_{j}^{i}\right\} \in \mathscr{D}^{k}$ be the sparse family. Then given $x \in \Omega_{i} \backslash \Omega_{i+1}$, there exists $Q_{j}^{i}$ such that

$$
M f(x) \leq a^{i+1}<a f_{Q_{j}^{i}} f(y) d \mu(y)
$$

hence, for $\mu$-a.e. $x$,

$$
M^{k} f(x) \leq a \sum_{i, j} f_{Q_{j}^{i}} f(y) d \mu(y) \cdot \chi_{Q_{j}^{i}}(x)=a T^{S_{k}} f(x) .
$$

If we now combine inequalities (3.4) and (3.5), we have that

$$
I_{1} \leq C(T, X)\|M f\|_{Y} \leq C(T, X) \sum_{k=1}^{K}\left\|T^{S_{k}} f\right\|_{Y} \leq C(T, X) \sup _{\mathscr{D}, S}\left\|T^{S} f\right\|_{Y} .
$$

To estimate $I_{2}$ we will decompose each $A_{i} f$ and apply duality. By $[\mathbf{1 7}$, Theorem 4.1] there exists a family of dyadic grids $\mathscr{D}^{1}, \ldots, \mathscr{D}^{J}$, satisfying the properties of Theorem 2.1 with the additional property that given any ball $\mathscr{B}_{\rho}$, there exists $j$ and $Q^{*} \subset \mathscr{D}^{j}$ such that $\mathscr{B}_{\rho} \subset Q^{*}$ and $\mu\left(\mathscr{B}_{\rho}\right) \approx$ $\mu\left(Q^{*}\right)$, with constants depending only on $X$. Recall (see equation (2.1) and the discussion after Theorem 2.1) that $2^{i} Q$ is defined to be a ball. Therefore, if we define

$$
S_{N}^{j}=\left\{Q \in S_{N}: \exists Q^{*} \in \mathscr{D}^{j}, 2^{i} Q \subset Q^{*}\right\},
$$

then

$$
A_{i} f(x) \leq C(X) \sum_{j=1}^{J} \sum_{\substack{Q \in S_{N}^{j} \\ 2^{i} Q \subset Q^{*}}} f_{Q^{*}} f(y) d \mu(y) \cdot \chi_{Q}(x)=C(X) \sum_{j=1}^{J} B_{i, j} f(x) .
$$

Arguing as in [2, Section 6] (see especially Lemmas 6.5 and 6.13) we apply the same argument used to prove (3.3) for $T$ to the adjoint operators $B_{i, j}^{*}$. Key to this is the fact that adjoint operators are weak $(1,1)$ with a constant that is linear in $i$. This yields the following pointwise estimate:

$$
\begin{array}{r}
B_{i, j}^{*} f(x) \leq i C_{1}(X) M f(x)+i C_{2}(X) \sum_{Q \in S_{*}^{j}} f_{Q} f(y) d \mu(y) \cdot \chi_{Q}(x) \\
=i C_{1}(X) M f(x)+i C_{2}(X) T_{i, j} f(x),
\end{array}
$$

where $S_{*}^{j} \subset \mathscr{D}^{j}$ is sparse. Therefore, repeating the above argument for bounding the maximal operator, we have that $B_{i, j}^{*}$ is bounded pointwise 
by a finite sum of sparse operators $T^{S^{l}}, 1 \leq l \leq L$ (defined with respect to a finite collection of dyadic grids $\mathscr{D}$ ). We can now estimate $I_{2}$ by duality using the fact that the operators $T^{S^{l}}$ are self-adjoint: there exists a collection of $g_{i} \in Y^{\prime},\left\|g_{i}\right\|_{Y^{\prime}}=1$, such that

$$
\begin{aligned}
I_{2} & =C(T, X) \sum_{i} 2^{-i \eta}\left\|A_{i} f \cdot \chi_{Q_{N}}\right\|_{Y} \\
& =C(T, X) \sum_{i} 2^{-i \eta} \int_{Q_{N}} A_{i} f(x) g_{i}(x) d \mu(x) \\
& \leq C(T, X) \sum_{i} 2^{-i \eta} \sum_{j=1}^{J} \int_{Q_{N}} B_{i, j} f(x) g_{i}(x) d \mu(x) \\
& \leq C(T, X) \sum_{i} 2^{-i \eta} \sum_{j=1}^{J} \int_{X} f(x) B_{i, j}^{*} g_{i}(x) d \mu(x) \\
& \leq C(T, X) \sum_{i} i 2^{-i \eta} \sum_{j=1}^{J} \sum_{l=1}^{L} \int_{X} f(x) T^{S^{l}} g_{i}(x) d \mu(x) \\
& =C(T, X) \sum_{i} i 2^{-i \eta} \sum_{j=1}^{J} \sum_{l=1}^{L} \int_{X} T^{S^{l}} f(x) g_{i}(x) d \mu(x) \\
& \leq C(T, X) \sum_{i} i 2^{-i \eta} \sum_{j=1}^{J} \sum_{l=1}^{L}\left\|T^{S^{l}} f\right\|_{Y}\left\|g_{i}\right\|_{Y^{\prime}} \\
& \leq C(T, X) \sup _{\mathscr{D}, S}\left\|T^{S} f\right\|_{Y} .
\end{aligned}
$$

This completes the proof of Theorem 3.1.

\section{Proof of Theorem 1.4}

We will prove this result for sparse operators, with the $[u, \sigma]_{A, B, p}$ condition replaced by the $[u, \sigma]_{A, B, p}^{\mathscr{D}}$ condition. Theorem 1.4 then follows immediately by Theorem 3.2 and Lemma 2.3. The proof for sparse operators is essentially identical to the proof in the Euclidean case in $[\mathbf{9}]$; for the convenience of the reader we sketch the details.

We need one preliminary result. In the Euclidean case this is due to Pérez [32], and in an SHT to Pérez and Wheeden [33], Pradolini and Salinas [34], and the first author [1]. In the latter papers the proofs are for maximal operators defined with respect to balls instead of dyadic cubes, but the proofs rely on a version of Theorem 2.8 for balls and so immediately adapt to this setting. 
Lemma 4.1. Given an $S H T(X, \rho, \mu)$ and a Young function $\Phi$ such that $\Phi \in B_{p}$, then

$$
\left\|M_{\Phi}^{\mathscr{D}} f\right\|_{L^{p}(\mu)} \leq C(X)[\Phi]_{B_{p}}^{1 / p}\|f\|_{L^{p}(\mu)} .
$$

Remark 4.2. In $[\mathbf{3 2}, \mathbf{3 4}]$ it is assumed that $\Phi$ satisfies the doubling condition $\Phi(2 t) \leq C \Phi(t)$. However, as noted in [10, p. 102] this assumption is only used to prove an equivalent formulation of the $B_{p}$ condition and can be removed. In the Euclidean case this is due to Liu and Luque [24]. In recent paper $[\mathbf{1}]$ the first author has shown that the doubling assumption can be fully removed in SHT.

Proof of Theorem 1.4: By duality and the definition of $T^{S}$, there exists $g \in L^{p^{\prime}}(u),\|g\|_{L^{p^{\prime}}(u)}=1$, such that

$$
\begin{aligned}
\left\|T^{S}(f \sigma)\right\|_{L^{p}(u)} & =\int_{X} T^{S}(f \sigma)(x) u(x) g(x) d \mu(x) \\
& \leq 2 \sum_{Q \in S} f_{Q} f(x) \sigma(x) d \mu(x) f_{Q} u(x) g(x) d \mu(x) \cdot \mu(E(Q)) \\
& \leq 8 \sum_{Q \in S}\left\|f \sigma^{1 / p}\right\|_{\bar{B}, Q}\left\|g u^{1 / p^{\prime}}\right\|_{\bar{A}, Q}\left\|u^{1 / p}\right\|_{A, Q}\left\|\sigma^{1 / p^{\prime}}\right\|_{B, Q} \mu(E(Q)) \\
& \leq 8[u, \sigma]_{A, B, p}^{\mathscr{D}} \int_{X} M_{\bar{B}}^{\mathscr{D}}\left(f \sigma^{1 / p}\right)(x) M_{\bar{A}}^{\mathscr{D}}\left(g u^{1 / p^{\prime}}\right)(x) d \mu(x) \\
& \leq 8[u, \sigma]_{A, B, p}^{\mathscr{D}}\left\|M_{\bar{B}}^{\mathscr{D}}\left(f \sigma^{1 / p}\right)\right\|_{p}\left\|M_{\bar{A}}^{\mathscr{D}}\left(g u^{1 / p^{\prime}}\right)\right\|_{p^{\prime}} \\
& \leq C(X)[u, \sigma]_{A, B, p}^{\mathscr{D}}[\bar{A}]_{B_{p^{\prime}}}^{1 / p^{\prime}}[\bar{B}]_{B_{p}}^{1 / p}\|f\|_{L^{p}(\sigma)}\|g\|_{L^{p^{\prime}}(u)}
\end{aligned}
$$

In the next section we will need an equivalent version of this result for sparse operators, and so we state it here. The equivalence is easily seen by letting $\sigma=v^{1-p^{\prime}}$.

Theorem 4.3. Given an $\operatorname{SHT}(X, \rho, \mu)$, a dyadic grid $\mathscr{D}$, a sparse family $S \subset \mathscr{D}$, and Young functions $A, B$, with $\bar{A} \in B_{p^{\prime}}$ and $\bar{B} \in B_{p}$, suppose the pair of weights $(u, v)$ satisfies

$$
[[u, v]]_{A, B, p}^{\mathscr{D}}=\sup _{Q \in \mathscr{D}}\left\|u^{1 / p}\right\|_{A, Q}\left\|v^{-1 / p}\right\|_{B, Q}<\infty .
$$

Then

$$
\left\|T^{S} f\right\|_{L^{p}(u)} \leq C(X)[[u, v]]_{A, B, p}^{\mathscr{D}}[\bar{A}]_{B_{p^{\prime}}}^{1 / p^{\prime}}[\bar{B}]_{B_{p}}^{1 / p}\|f\|_{L^{p}(v)}
$$




\section{A weak $(1,1)$ inequality}

In this section we prove a two-weight, weak $(1,1)$ inequality for sparse operators. A version of this result for general Calderón-Zygmund operators in the Euclidean case was due to Pérez $[\mathbf{3 1}]$ and our proof closely follows his. However, it is simplified because we are working with sparse operators: instead of appealing to duality and the Coifman-Fefferman inequality relating singular integrals and the maximal operator, we use two-weight theory via Theorem 4.3.

Theorem 5.1. Given an $S H T(X, \rho, \mu)$, let $\mathscr{D}$ be a dyadic grid satisfying the hypotheses of Theorem 2.1, and let $S \subset \mathscr{D}$ be sparse. Let $\Phi$ be a Young function such that for some $1<q<\infty, A_{\Phi}(t)=\Phi\left(t^{q}\right)$ satisfies $\bar{A}_{\Phi} \in B_{q^{\prime}}$. Then for all $\lambda>0$ and $f \geq 0$

$$
u\left(\left\{x \in X: T^{S} f(x)>\lambda\right\}\right) \leq C(X, \Phi, q) \frac{1}{\lambda} \int_{X} f(x) M_{\Phi}^{\mathscr{D}} u(x) d \mu(x) .
$$

Proof: We first consider the case when $\mu(X)=\infty$; at the end of the proof we will sketch the changes needed when $\mu(X)<\infty$.

Fix $\lambda>0$ and let the disjoint cubes $\left\{Q_{j}\right\}$ and functions $g$ and $b=$ $\sum_{j} b_{j}$ be as given by Theorem 2.9. Since $f=g+b$, we have that

$$
\begin{aligned}
u( & \left.\left\{x \in X: T^{S} f(x)>\lambda\right\}\right) \\
& =u(\Omega)+u\left(\left\{x \in \Omega^{c}:\left|T^{S} b(x)\right|>\lambda / 2\right\}\right)+u\left(\left\{x \in \Omega^{c}: T^{S} g(x)>\lambda / 2\right\}\right) \\
& =I_{1}+I_{2}+I_{3},
\end{aligned}
$$

where $\Omega=\bigcup_{j} Q_{j}$.

The estimate for $I_{1}$ is immediate: since $\mu$ is doubling, by the properties of the cubes $\left\{Q_{j}\right\}$,

$$
\begin{aligned}
I_{1}= & u(\Omega) \leq \sum_{j} u\left(Q_{j}\right)=\sum_{j} \frac{u\left(Q_{j}\right)}{\mu\left(Q_{j}\right)} \mu\left(Q_{j}\right) \leq \frac{1}{\lambda} \sum_{j} \frac{u\left(Q_{j}\right)}{\mu\left(Q_{j}\right)} \int_{Q_{j}} f(x) d \mu(x) \\
& \leq \frac{1}{\lambda} \sum_{j} \int_{Q_{j}} f(x) M^{\mathscr{D}} u(x) d \mu(x) \leq C(\Phi) \frac{1}{\lambda} \int_{X} f(x) M_{\Phi}^{\mathscr{D}} u(x) d \mu(x) ;
\end{aligned}
$$

the last inequality follows from the fact that since $t \lesssim \Phi(t),\|u\|_{1, Q} \leq$ $C(\Phi)\|u\|_{\Phi, Q}$. 
To estimate $I_{2}$, fix $x \in \Omega^{c}$; then $x \in Q_{j}^{c}$ for all $j$. By linearity, $T^{S} b(x)=\sum_{j} T^{S} b_{j}(x)$, and for each $j$,

$$
\begin{aligned}
T^{S} b_{j}(x) & =\sum_{Q \in S} f_{Q} b_{j}(y) d \mu(y) \cdot \chi_{Q}(x) \\
& =\sum_{Q \in S} \frac{\mu\left(Q_{j}\right)}{\mu(Q)} \frac{1}{\mu\left(Q_{j}\right)} \int_{Q}\left(f(y)-f_{Q_{j}}\right) \chi_{Q_{j}}(y) d \mu(y) \cdot \chi_{Q}(x) .
\end{aligned}
$$

For $T^{S} b_{j}(x) \neq 0$, we need $x \in Q$, which in turn implies that $Q \cap Q_{j} \neq \emptyset$ and $Q \cap Q_{j}^{c} \neq \emptyset$. Since $Q, Q_{j} \in \mathscr{D}$, we must have that $Q_{j} \subset Q$. But then

$$
\frac{1}{\mu\left(Q_{j}\right)} \int_{Q}\left(f(y)-f_{Q_{j}}\right) \chi_{Q_{j}}(y) d \mu(y)=\frac{1}{\mu\left(Q_{j}\right)} \int_{Q_{j}}\left(f(y)-f_{Q_{j}}\right) d \mu(y)=0 .
$$

Hence, $T^{S} b_{j}(x)=0$ and so $I_{2}=0$.

To estimate $I_{3}$ we want to apply Theorem 4.3 with the pair $\left(u, M_{\Phi} u\right)$. Let $B(t)=t^{(r q)^{\prime}}$ with $1 / q<r<1$; then $\bar{B} \in B_{q}$ and $[\bar{B}]_{B_{q}} \leq C(q)$. We claim that

$$
\left[\left[u, M_{\Phi}^{\mathscr{D}} u\right]\right]_{A_{\Phi}, B, p}^{\mathscr{D}} \leq 1 .
$$

To see this, fix $Q \in \mathscr{D}$. Since $B(1)=1$, it follows that $\left\|\chi_{Q}\right\|_{B, Q}=1$. Moreover, for any $x \in Q$, by a change of variables in the definition of the Orlicz norm,

$$
M_{\Phi}^{\mathscr{D}} u(x) \geq\|u\|_{\Phi, Q}=\left\|u^{1 / q}\right\|_{A_{\Phi}, Q}^{q} .
$$

Therefore,

$$
\left\|u^{1 / p}\right\|_{A_{\Phi}, Q}\left\|M_{\Phi}^{\mathscr{Q}}(u)^{-1 / p}\right\|_{B, Q} \leq\left\|u^{1 / p}\right\|_{A_{\Phi}, Q}\left\|u^{1 / p}\right\|_{A_{\Phi}, Q}^{-1}\left\|\chi_{Q}\right\|_{B, Q}=1 .
$$


Hence, by Theorem 4.3 and since $g(x) \leq C(X) \lambda$,

$$
\begin{aligned}
I_{3} \leq & \frac{2^{q}}{\lambda^{q}} \int_{\Omega^{c}} T^{S} g(x)^{q} u(x) d \mu(x) \\
\leq & C(X, \Phi, q) \frac{1}{\lambda^{q}} \int_{X} g(x)^{q} M_{\Phi}^{\mathscr{O}}\left(u \chi_{\Omega^{c}}\right)(x) d \mu(x) \\
\leq & C(X, \Phi, q) \frac{1}{\lambda} \int_{X} g(x) M_{\Phi}^{\mathscr{D}}\left(u \chi_{\Omega^{c}}\right)(x) d \mu(x) \\
= & C(X, \Phi, q) \frac{1}{\lambda} \int_{\Omega^{c}} f(x) M_{\Phi}^{\mathscr{D}}\left(u \chi_{\Omega^{c}}\right) d \mu(x) \\
& +C(X, \Phi, q) \frac{1}{\lambda} \sum_{j} \int_{Q_{j}} f_{Q_{j}} M_{\Phi}^{\mathscr{O}}\left(u \chi_{\Omega^{c}}\right)(x) d \mu(x) \\
= & J_{1}+J_{2} .
\end{aligned}
$$

Clearly,

$$
J_{1} \leq C(X, \Phi, q) \frac{1}{\lambda} \int_{X} f(x) M_{\Phi}^{\mathscr{D}} u(x) d \mu(x),
$$

as desired. To estimate $J_{2}$, assume for the moment that for each $j$ and $x \in Q_{j}$,

$$
M_{\Phi}^{\mathscr{D}}\left(u \chi_{\Omega^{c}}\right)(x) \leq \inf _{y \in Q_{j}} M_{\Phi}^{\mathscr{D}}\left(u \chi_{Q_{j}^{c}}\right)(y) .
$$

Given this, we have that

$$
\begin{aligned}
J_{2} & \leq C(X, \Phi, q) \frac{1}{\lambda} \sum_{j} f_{Q_{j}} \mu\left(Q_{j}\right) \inf _{y \in Q_{J}} M_{\Phi}^{D}\left(u \chi_{Q_{j}^{c}}\right)(y) \\
& \leq C(X, \Phi, q) \frac{1}{\lambda} \sum_{j} \int_{Q_{j}} f(y) M_{\Phi}^{\mathscr{D}}\left(u \chi_{Q_{j}^{c}}\right)(y) d \mu(y) \\
& \leq C(X, \Phi, q) \frac{1}{\lambda} \int_{X} f(y) M_{\Phi}^{\mathscr{D}} u(y) d \mu(y) .
\end{aligned}
$$

It remains to prove (5.2). But if $x \in Q_{j}$, then

$$
M_{\Phi}^{\mathscr{D}}\left(u \chi_{\Omega^{c}}\right)(x) \leq M_{\Phi}^{\mathscr{O}}\left(u \chi_{Q_{j}^{c}}\right)(x)=\sup _{x \in Q \in \mathscr{D}}\left\|u \chi_{Q_{j}^{c}}\right\|_{\Phi, Q} .
$$

The norm on the right hand side is non-zero only if $x \in Q$ and $Q \cap Q_{j}^{c} \neq \emptyset$. Therefore, by the properties of dyadic cubes we must have that $Q \subset Q_{j}$. 
Hence,

$$
M_{\Phi}^{\mathscr{D}}\left(u \chi_{Q_{j}^{c}}\right)(x)=\sup _{\substack{Q \in \mathscr{D} \\ Q_{j} \subset Q}}\left\|u \chi_{Q_{j}^{c}}\right\|_{\Phi, Q},
$$

and since this quantity is independent of $x \in Q_{j}$, we get (5.2).

If $\mu(X)<\infty$, then we can repeat the above proof for all $\lambda>f_{X} f(x) d \mu(x)$. If the opposite inequality holds, then for some dyadic cube $Q$ sufficiently large, $Q=X$, and so

$$
\begin{aligned}
u(\{x & \left.\left.\in X: T^{S} f(x)>\lambda\right\}\right) \leq u(X) \leq \frac{u(Q)}{\mu(Q)} \frac{1}{\lambda} \int_{Q} f(x) d \mu(x) \\
& \leq \frac{1}{\lambda} \int_{Q} f(x) M^{\mathscr{D}} u(x) d \mu(x) \leq C(\Phi) \frac{1}{\lambda} \int_{Q} f(x) M_{\Phi}^{\mathscr{D}} u(x) d \mu(x) .
\end{aligned}
$$

\section{Proof of Theorems $\mathbf{1 . 5}$ and $\mathbf{1 . 6}$}

We first show that Theorem 1.6 is a consequence of Theorem 1.5. Given both separated bump conditions, the latter result implies that

$$
\begin{aligned}
\left\|T^{S}(\cdot \sigma)\right\|_{L^{p}(\sigma) \rightarrow L^{p, \infty}(u)} & \lesssim[u, \sigma]_{A, p}, \\
\left\|T^{S}(\cdot u)\right\|_{L^{p^{\prime}}(u) \rightarrow L^{p^{\prime}, \infty}(\sigma)} & \lesssim[\sigma, u]_{B, p^{\prime}} .
\end{aligned}
$$

Therefore, by Theorem 3.2 we get the desired strong type inequality.

To prove Theorem 1.5 it will again suffice to prove it for sparse operators. In order to do this we need a weighted norm inequality for an Orlicz maximal operator. The following result was proved in $[\mathbf{1 1}]$ for the Hardy-Littlewood maximal operator in the Euclidean case; the proof in an SHT is nearly the same and we sketch the details.

Lemma 6.1. Given $1<p<\infty$, let $A, C$, and $\Phi$ be Young functions such that $A^{-1}(t) C^{-1}(t) \leq c_{0} \Phi^{-1}(t)$ for $t>0$ where and $C \in B_{p^{\prime}}$. If $[u, \sigma]_{A, p}^{\mathscr{D}}<\infty$, then

$$
\left\|M_{\Phi}^{\mathscr{D}}(f u)\right\|_{L^{p^{\prime}}(\sigma)} \leq C\left(X, c_{0}\right)[u, \sigma]_{A, p}[C]_{B_{p^{\prime}}}^{1 / p^{\prime}}\|f\|_{L^{p^{\prime}}(u)} .
$$

Proof: We first consider the case when $\mu(X)=\infty$. By Theorem 2.8, fix $a>1$ sufficiently large and form the cubes $\left\{Q_{j}^{k}\right\}$ such that

$$
\Omega_{k}=\left\{x: M_{\Phi}^{\mathscr{D}}(f u)(x)>a^{k}\right\}=\bigcup_{j} Q_{j}^{k} .
$$


Then by the generalized Hölder's inequality we have that

$$
\begin{aligned}
\int_{X} M_{\Phi}^{\mathscr{D}}(f u) & (x)^{p^{\prime}} \sigma(x) d \mu(x) \\
& =\sum_{k} \int_{\Omega_{k} \backslash \Omega_{k+1}} M_{\Phi}^{\mathscr{D}}(f u)(x)^{p^{\prime}} \sigma(x) d \mu(x) \\
& \leq a^{p^{\prime}} \sum_{k, j}\|f u\|_{\Phi, Q_{j}^{k}}^{p^{\prime}} \sigma\left(Q_{j}^{k}\right) \\
& \leq C\left(X, c_{0}\right) \sum_{k, j}\left\|f u^{1 / p^{\prime}}\right\|_{C, Q_{j}^{k}}^{p^{\prime}}\left\|u^{1 / p}\right\|_{A, Q_{j}^{k}}^{p^{\prime}}\left\|\sigma^{1 / p^{\prime}}\right\|_{p^{\prime}, Q_{j}^{k}}^{p^{k}} E\left(Q_{j}^{k}\right) \\
& \leq C\left(X, c_{0}\right)[u, \sigma]_{A, p}^{p^{\prime}} \sum_{k, j} \int_{E\left(Q_{j}^{k}\right)} M_{C}^{\mathscr{D}}\left(f u^{1 / p^{\prime}}(x)\right)^{p^{\prime}} d \mu(x) \\
& \leq C\left(X, c_{0}\right)[u, \sigma]_{A, p}^{p^{\prime}} \int_{X} M_{C}^{\mathscr{D}}\left(f u^{1 / p^{\prime}}(x)\right)^{p^{\prime}} d \mu(x) \\
& \leq C\left(X, c_{0}\right)[u, \sigma]_{A, p}^{p^{\prime}}[C]_{B_{p^{\prime}}} \int_{X} f(x)^{p^{\prime}} u(x) d \mu(x) .
\end{aligned}
$$

If $\mu(X)<\infty$, then let $k_{0}$ be the largest integer such that

$$
a^{k_{0}}<\|f\|_{X, \Phi}
$$

Then $\Omega_{k_{0}}=X$. We can repeat the above argument summing over $k \geq k_{0}$, and for $k>k_{0}$ we can still form the cubes $\left\{Q_{j}^{k}\right\}$ and argue as before. When $k=k_{0}$, then there exists a large dyadic cube $Q=X$. Hence, $a^{k_{0}}<\|f\|_{\Phi, Q}$ and the argument proceeds as before, replacing the collection $\left\{Q_{j}^{k}\right\}$ with the single cube $Q$.

We can now prove Theorem 1.5. This is the only part of the proof in which we use the fact that $A$ is a log-bump. Our proof relies on an openness property that is particular to log-bumps. More precisely, given $A(t)=t^{p} \log (e+t)^{p-1+\delta}$ where $\delta>0$, then $\bar{A} \in B_{p^{\prime}}$ and

$$
A^{-1}(t) \bar{A}^{-1}(t) \leq t \text {. }
$$

But in fact we can find $\epsilon>0$ and $C \in B_{p^{\prime}}$ such that

$$
A^{-1}(t) C^{-1}(t) \leq \frac{c t}{\log (e+t)^{\epsilon}} .
$$

For a different way to view this openness property, see Remark 10 and the preceding discussion in $[\mathbf{1 4}]$. A weaker version of this property would be 
sufficient (see [11, Theorem 4.1]) but it seems doubtful that this property is satisfied by an arbitrary bump function.

The proof uses an extrapolation argument from $[\mathbf{1 1}]$; see also $[\mathbf{1 0}$, Chapter 8]. Fix $\lambda>0$ and define

$$
\Omega_{\lambda}=\left\{x \in X: T^{S}(f \sigma)(x)>\lambda\right\} .
$$

Then by duality, there exists $h \in L^{p^{\prime}}(u),\|h\|_{L^{p^{\prime}(u)}}=1$, such that

$$
u\left(\Omega_{\lambda}\right)^{1 / p}=\left\|\chi_{\Omega_{\lambda}}\right\|_{L^{p}(u)}=\int_{\Omega_{\lambda}} u(x) h(x) d \mu(x)=(u h)\left(\Omega_{\lambda}\right) .
$$

Now let $\Phi(t)=t \log (e+t)^{\epsilon}$, where we will fix the value of $\epsilon>0$ below. Let $q-1=\epsilon / 2$ and let $A_{\Phi}(t)=t^{q} \log (e+t)^{q-1+\epsilon / 2}$. Then $\bar{A}_{\Phi} \in B_{q^{\prime}}$, and so by Theorem 5.1,

$$
\begin{aligned}
(u h)\left(\Omega_{\lambda}\right) & \leq C(X, \epsilon) \frac{1}{\lambda} \int_{X} f(x) \sigma(x) M_{\Phi}^{\mathscr{D}}(u h)(x) d \mu(x) \\
& \leq C(X, \epsilon) \frac{1}{\lambda}\|f\|_{L^{p}(\sigma)}\left\|M_{\Phi}^{\mathscr{D}}(u h)\right\|_{L^{p^{\prime}}(\sigma)} .
\end{aligned}
$$

Now fix $\epsilon<\delta / p$ and define $C(t)=t^{p^{\prime}} \log (e+t)^{-1-\left(p^{\prime}-1\right) \eta}$, where $\eta=\delta-\epsilon p$. Then $C \in B_{p^{\prime}}$ and $[C]_{B_{p^{\prime}}}$ depends only on $p$ and $\delta$. Moreover, we have that $A^{-1}(t) C^{-1}(t) \leq c_{0} \Phi^{-1}(t)$, where the constant $c_{0}$ depends on $\delta$ and $p$. (See $[\mathbf{1 1}]$ for details.) Therefore, by Lemma 6.1,

$$
\left\|M_{\Phi}^{\mathscr{D}}(h u)\right\|_{L^{p^{\prime}(\sigma)}} \leq C(X, p, \delta)[u, \sigma]_{A, p}\|h\|_{L^{p^{\prime}(u)}}=C(X, p, \delta)[u, \sigma]_{A, p} .
$$

Combining the above inequalities we get the desired result.

\section{Separated and double bump conditions}

We construct our example on the real line with $p=2$. Our example can be readily modified to work for other values of $p$. Define the Young functions

$$
A(t)=B(t)=t^{2} \log (e+t)^{2} .
$$

Then $\bar{A}, \bar{B} \in B_{2}$. By rescaling, if we let $\Phi(t)=t \log (e+t)^{2}$, then for any pair $(u, \sigma)$,

$$
\left\|u^{1 / 2}\right\|_{A, Q} \approx\|u\|_{\Phi, Q}^{1 / 2}, \quad\left\|\sigma^{1 / 2}\right\|_{B, Q} \approx\|\sigma\|_{\Phi, Q}^{1 / 2} .
$$

Therefore, it will suffice estimate the norms of $u$ and $\sigma$ with respect to $\Phi$. Similarly, we can replace the localized $L^{2}$ norms of $u^{1 / 2}$ and $\sigma^{1 / 2}$ with the $L^{1}$ norms of $u$ and $\sigma$.

Before we define $u$ and $\sigma$ we first construct a pair $\left(u_{0}, \sigma_{0}\right)$ which will be the basic building block for our example. Fix an integer $n \geq 2$ and define $Q=(0, n), \sigma_{0}=\chi_{(0,1)}$, and $u_{0}=K_{n} \chi_{(n-1, n)}$, where $K_{n}=$ 
$n^{2} \log (e+n)^{-3}$. Then a straightforward estimate with the definition of the Orlicz norm shows that

$$
\begin{aligned}
& \left\|u_{0}\right\|_{1, Q}=\frac{K_{n}}{n}, \quad\left\|u_{0}\right\|_{\Phi, Q} \approx \frac{K_{n} \log (e+n)^{2}}{n}, \\
& \left\|\sigma_{0}\right\|_{1, Q}=\frac{1}{n}, \quad\left\|\sigma_{0}\right\|_{\Phi, Q} \approx \frac{\log (e+n)^{2}}{n} .
\end{aligned}
$$

Therefore, we have that

$$
\left\|u_{0}\right\|_{1, Q}\left\|\sigma_{0}\right\|_{\Phi, Q}, \quad\left\|u_{0}\right\|_{\Phi, Q}\left\|\sigma_{0}\right\|_{1, Q} \approx \frac{1}{\log (e+n)},
$$

but

$$
\left\|u_{0}\right\|_{\Phi, Q}\left\|\sigma_{0}\right\|_{\Phi, Q} \approx \log (e+n) .
$$

We now define $u$ and $\sigma$ as follows:

$$
u(x)=\sum_{n \geq 2} K_{n} \chi_{I_{n}}(x) \quad \sigma(x)=\sum_{n \geq 2} \chi_{J_{n}}(x),
$$

where $I_{n}=\left(e^{n}+n-1, e^{n}+n\right)$ and $J_{n}=\left(e^{n}, e^{n}+1\right)$. Since the above computations are translation invariant, we immediately get that if $Q_{n}=\left(e^{n}, e^{n}+n\right)$, then

$$
\|u\|_{\Phi, Q_{n}}\|\sigma\|_{\Phi, Q_{n}} \approx \log (e+n),
$$

and so $[u, \sigma]_{A, B, 2}=\infty$.

It remains, therefore, to show that $[u, \sigma]_{A, 2}$ and $[\sigma, u]_{B, 2}$ are both finite. We will consider $[u, \sigma]_{A, 2}$; the argument for the second is essentially the same. Fix an interval $Q$; we will show that $\|u\|_{\Phi, Q}\|\sigma\|_{1, Q}$ is uniformly bounded. Fix an integer $N$ such that $N-1 \leq|Q| \leq N$. We need to consider those values of $n$ such that $Q$ intersects either $I_{n}$ or $J_{n}$.

Suppose that for some $n \geq N+2, Q$ intersects $I_{n}$. But in this case it cannot intersect $J_{k}$ for any $k$ and so $\|\sigma\|_{1, Q}=0$. Similarly, if $Q$ intersects $J_{n}$, then $\|u\|_{\Phi, Q}=0$.

Now suppose that for some $n<N+2, Q$ intersects one of $I_{n}$ or $J_{n}$. If $\log (N) \lesssim n$ (more precisely, if $N<e^{n}-e^{n-1}-1$ ), then for any $k \neq n$, $Q$ cannot intersect $I_{k}$ or $J_{k}$. In this case $\|u\|_{\Phi, Q}\|\sigma\|_{1, Q} \neq 0$ only if $Q$ intersects both $I_{n}$ and $J_{n}$, and will reach its maximum when $N \approx n$. But in this case we can replace $Q$ by $\left(e^{n}, e^{n}+n\right)$ and the above computation shows that $\|u\|_{\Phi, Q}\|\sigma\|_{1, Q} \lesssim 1$.

Finally, suppose $Q$ intersects one or more pairs $I_{n}$ and $J_{n}$ with $n \lesssim$ $\log (N)$. Then $|\operatorname{supp}(u) \cap Q| \lesssim \log (N)$ and $\|u\|_{L^{\infty}(Q)} \approx K_{\lfloor\log (N)\rfloor} \lesssim$ 
$\log (N)^{2}$. Therefore,

$$
\|u\|_{\Phi, Q} \lesssim\|u\|_{2, Q} \leq\|u\|_{L^{\infty}(Q)}\left(\frac{|\operatorname{supp}(u) \cap Q|}{|Q|}\right)^{1 / 2} \lesssim \frac{\log (N)^{5 / 2}}{N^{1 / 2}} .
$$

A similar calculation shows that

$$
\|\sigma\|_{1, Q} \lesssim \frac{\log (N)}{N}
$$

hence, we again have that $\|u\|_{\Phi, Q}\|\sigma\|_{1, Q} \lesssim 1$. It follows that $[u, \sigma]_{A, 2}<$ $\infty$ and our proof is complete.

Remark 7.1. If we modify our example by defining $K_{n}=n^{2} \log (e+n)^{-2}$, then the same arguments show that the pair $(u, \sigma)$ satisfies the separated bump condition when $A(t)=B(t)=t^{2} \log (e+t)^{1+\delta}, 0<\delta<2$, but does not satisfy the double bump condition for any $\delta>0$. It would be of interest to construct a pair that satisfies a separated bump condition for some pair of $\log$ bumps but fails to satisfy the double bump condition for any pair of Young functions for which the appropriate $B_{p}$ conditions hold.

\section{References}

[1] T. C. Anderson, A new sufficient two-weighted bump assumption for $L^{p}$ boundedness of Calderón-Zygmund operators, Preprint (2012).

[2] T. C. Anderson and A. Vagharshakyan, A simple proof of the sharp weighted estimate for Calderón-Zygmund operators on homogeneous spaces, J. Geom. Anal. 24(3) (2014), 1276-1297. DOI: $10.1007 / \mathrm{s} 12220-012-9372-7$.

[3] A. L. Bernardis, G. Pradolini, M. Lorente, and M. S. RiVeros, Composition of fractional Orlicz maximal operators and $A_{1}$-weights on spaces of homogeneous type, Acta Math. Sin. (Engl. Ser.) 26(8) (2010), 1509-1518. DOI: 10.1007/s10114-010-8445-4.

[4] A.-P. CALderón, Inequalities for the maximal function relative to a metric, Studia Math. 57(3) (1976), 297-306.

[5] M. Christ, "Lectures on singular integral operators", CBMS Regional Conference Series in Mathematics 77, Published for the Conference Board of the Mathematical Sciences, Washington, DC; by the American Mathematical Society, Providence, RI, 1990.

[6] R. R. Coifman and G. Weiss, "Analyse harmonique non-commutative sur certains espaces homogènes", Étude de certaines intégrales singulières, Lecture Notes in Mathematics 242, Springer-Verlag, Berlin-New York, 1971. 
[7] R. R. Coifman and G. Weiss, Extensions of Hardy spaces and their use in analysis, Bull. Amer. Math. Soc. 83(4) (1977), 569-645. DOI: $10.1090 /$ S0002-9904-1977-14325-5.

[8] D. Cruz-Uribe, J. M. Martell, and C. Pérez, Sharp twoweight inequalities for singular integrals, with applications to the Hilbert transform and the Sarason conjecture, Adv. Math. 216(2) (2007), 647-676. DOI: 10.1016/j.aim.2007.05.022.

[9] D. Cruz-Uribe, J. M. Martell, and C. PÉrez, Sharp weighted estimates for classical operators, Adv. Math. 229(1) (2012), 408-441. DOI: 10.1016/j.aim.2011.08.013.

[10] D. V. Cruz-Uribe, J. M. Martell, and C. PÉrez, "Weights, extrapolation and the theory of Rubio de Francia", Operator Theory: Advances and Applications 215, Birkhäuser/Springer Basel AG, Basel, 2011. DOI: 10.1007/978-3-0348-0072-3.

[11] D. Cruz-Uribe and C. PÉrez, Sharp two-weight, weak-type norm inequalities for singular integral operators, Math. Res. Lett. 6(4) (1999), 417-427. DOI: 10.4310/MRL.1999.v6.n4.a4.

[12] D. Cruz-Uribe and C. PÉrez, Two-weight, weak-type norm inequalities for fractional integrals, Calderón-Zygmund operators and commutators, Indiana Univ. Math. J. 49(2) (2000), 697-721. DOI: 10.1512/iumj . 2000.49.1795.

[13] D. Cruz-Uribe and C. PÉrez, On the two-weight problem for singular integral operators, Ann. Sc. Norm. Super. Pisa Cl. Sci. (5) 1(4) (2002), 821-849.

[14] D. Cruz-Uribe, A. Reznikov, and A. Volberg, Logarithmic bump conditions and the two-weight boundedness of Calderón-Zygmund operators, Adv. Math. 255 (2014), 706-729. DOI: 10.1016/j. aim. 2014.01.016.

[15] I. Genebashvili, A. Gogatishvili, V. Kokilashvili, and M. KRBEC, "Weight theory for integral transforms on spaces of homogeneous type", Pitman Monographs and Surveys in Pure and Applied Mathematics 92, Longman, Harlow, 1998.

[16] T. P. Hytönen, The $A_{2}$ theorem: Remarks and complements, Preprint (2012), arXiv:1212.3840v1.

[17] T. Hytönen and A. Kairema, Systems of dyadic cubes in a doubling metric space, Colloq. Math. 126(1) (2012), 1-33. DOI: 10.4064/cm126-1-1.

[18] T. Hytönen and A. Kairema, What is a cube? Ann. Acad. Sci. Fenn. Math. 38(2) (2013), 405-412. DOI: 10.5186/aasfm. 2013.3838. 
[19] T. P. Hytönen And M. T. LACEy, The $A_{p}-A_{\infty}$ inequality for general Calderón-Zygmund operators, Indiana Univ. Math. J. 61(6) (2012), 2041-2052. DOI : 10.1512/iumj .2012.61.4777.

[20] T. Hytönen And C. PÉrez, $L(\log L)^{\epsilon}, A_{1}$ and two weights for maximal singular integrals, Preprint (2013).

[21] T. Hytönen And C. PÉrez, Sharp weighted bounds involving $A_{\infty}$, Anal. PDE 6(4) (2013), 777-818. DOI : 10.2140/apde.2013. 6.777 .

[22] M. Lacey, E. T. SAwyer, and I. Uriarte-Tuero, Two weight inequalities for discrete positive operators, Preprint (2012).

[23] A. K. LERNER, On an estimate of Calderón-Zygmund operators by dyadic positive operators, J. Anal. Math. 121 (2013), 141-161. DOI: $10.1007 / \mathrm{s} 11854-013-0030-1$.

[24] L. LiU And T. Luque, A $B_{p}$ condition for the strong maximal function, Trans. Amer. Math. Soc. 366(11) (2014), 5707-5726. DOI: 10.1090/S0002-9947-2014-05956-4.

[25] R. A. Macías and C. Segovia, Lipschitz functions on spaces of homogeneous type, Adv. in Math. 33(3) (1979), 257-270. DOI: 10. 1016/0001-8708(79)90012-4.

[26] R. A. Macías And C. Segovia, A well behaved quasi-distance for spaces of homogeneous type, Trabajos de Matemática 32, Instituto Argentino de Matemática (IAM) (1980), 1-18. Available at http://www . cimec.org.ar.

[27] J. M. MARTELL, Sharp maximal functions associated with approximations of the identity in spaces of homogeneous type and applications, Studia Math. 161(2) (2004), 113-145. DOI: 10.4064/sm161 $-2-2$.

[28] F. Nazarov, A. Reznikov, S. Treil, and A. Volberg, A Bellman function proof of the $L^{2}$ bump conjecture, J. Anal. Math. 121(1) (2013), 255-277. DOI: 10.1007/s11854-013-0035-9.

[29] F. Nazarov, A. Reznikov, and A. Volberg, Bellman approach to the one-sided bumping for weighted estimates of Calderón-Zygmund operators, Preprint (2013), arXiv:1306.2653v1.

[30] C. PÉrez, Two weighted inequalities for potential and fractional type maximal operators, Indiana Univ. Math. J. 43(2) (1994), 663-683. DOI: 10.1512/iumj.1994.43.43028.

[31] C. PÉREz, Weighted norm inequalities for singular integral operators, J. London Math. Soc. (2) 49(2) (1994), 296-308. DOI: 10.1112/jlms/49.2.296.

[32] C. PÉrEz, On sufficient conditions for the boundedness of the Hardy-Littlewood maximal operator between weighted $L^{p}$-spaces 
with different weights, Proc. London Math. Soc. (3) 71(1) (1995), 135-157. DOI: 10.1112/plms/s3-71.1.135.

[33] C. Pérez and R. L. Wheeden, Uncertainty principle estimates for vector fields, J. Funct. Anal. 181(1) (2001), 146-188. DOI: 10.1006/jfan.2000.3711.

[34] G. Pradolini and O. Salinas, Maximal operators on spaces of homogeneous type, Proc. Amer. Math. Soc. 132(2) (2004), 435-441 (electronic). DOI : 10.1090/S0002-9939-03-07079-5.

[35] M. M. RaO And Z. D. Ren, "Theory of Orlicz spaces", Monographs and Textbooks in Pure and Applied Mathematics 146, Marcel Dekker, Inc., New York, 1991.

[36] W. Rudin, "Real and complex analysis", Third edition, McGrawHill Book Co., New York, 1987.

[37] R. Toledano, A note on the Lebesgue differentiation theorem in spaces of homogeneous type, Real Anal. Exchange 29(1) (2003/04), $335-339$.

[38] S. TreIL, A remark on two weight estimates for positive dyadic operators, Preprint (2012), arXiv:1201.1455v3.

[39] D. YANG, T1 theorems on Besov and Triebel-Lizorkin spaces on spaces of homogeneous type and their applications, Z. Anal. Anwendungen 22(1) (2003), 53-72. DOI: 10.4171/ZAA/1132.

Theresa C. Anderson:

Department of Mathematics

Brown University

Providence, RI 02912

USA

E-mail address: theresa_anderson@brown.edu

David Cruz-Uribe, SFO:

Department of Mathematics

Trinity College

Hartford, CT 06106

USA

E-mail address: david.cruzuribe@trincoll.edu

Kabe Moen:

Department of Mathematics

University of Alabama

Tuscaloosa, AL 35487

USA

E-mail address: kabe.moen@ua.edu

Primera versió rebuda el 26 d'agost de 2013, darrera versió rebuda el 15 de novembre de 2013. 